\title{
RNA-Seq De Novo Assembly and Differential Transcriptome Analysis of Korean Medicinal Herb Cirsium japonicum var. spinossimum
}

\author{
Neha Samir Roy ${ }^{1,2}$, Jung-A Kim ${ }^{3}$, Ah-Young Choi ${ }^{4}$, Yong-Wook Ban ${ }^{5}$, Nam-Il Park ${ }^{6}$, \\ Kyong-Cheul Park ${ }^{1}$, Hee-sun Yang ${ }^{3}$, Ik-Young Choi ${ }^{1,2}$, Soonok Kim ${ }^{3 * *}$ \\ ${ }^{1}$ Department of Agriculture and Life Industry, Kangwon National University, Chuncheon 24341, Korea, ${ }^{2}$ Agriculture and Life \\ Sciences Research Institute, Kangwon National University, Chuncheon 24341, Korea, ${ }^{3}$ Biological Resources Assessment Division, \\ National Institute of Biological Resources, Incheon 22689, Korea, ${ }^{4}$ DNACare Genomics Institute, Seongnam 13558, Korea, \\ ${ }^{5}$ Department of Forest Environmental System, Kangwon National University, Chuncheon 24341, Korea, ${ }^{6}$ Department of Plant \\ Science, Gangneung Wonju National University, Gangneung 25457, Korea
}

Cirsium japonicum belongs to the Asteraceae or Compositae family and is a medicinal plant in Asia that has a variety of effects, including tumour inhibition, improved immunity with flavones, and antidiabetic and hepatoprotective effects. Silymarin is synthesized by 4-coumaroyl-CoA via both the flavonoid and phenylpropanoid pathways to produce the immediate precursors taxifolin and coniferyl alcohol. Then, the oxidative radicalization of taxifolin and coniferyl alcohol produces silymarin. We identified the expression of genes related to the synthesis of silymarin in $C$. japonicum in three different tissues, namely, flowers, leaves, and roots, through RNA sequencing. We obtained 51,133 unigenes from transcriptome sequencing by de novo assembly using Trinity v2.1.1, TransDecoder v2.0.1, and CD-HIT v4.6 software. The differentially expressed gene analysis revealed that the expression of genes related to the flavonoid pathway was higher in the flowers, whereas the phenylpropanoid pathway was more highly expressed in the roots. In this study, we established a global transcriptome dataset for $C$. japonicum. The data shall not only be useful to focus more deeply on the genes related to product medicinal metabolite including flavolignan but also to study the functional genomics for genetic engineering of C. japonicum.

Keywords: Cirsium japonicum, differentially expressed gene, RNA-sequencing, silymarin, transcriptome

\section{Introduction}

Cirsium japonicum is a wild perennial herb found in many areas of Korea, Japan, and China. C. japonicum is used as an anti-haemorrhagic, anti-hypertensive and uretic agent in traditional Chinese medicine [1]. In traditional medicine, $C$. japonicum is sometimes used for the management of different types of cancer, including liver and uterine cancer and leukaemia [2]. To date, many studies have explored the effects of C. japonicum on various diseases. However, no reports have performed molecular biology studies or investigated comprehensive genomic and transcriptomic data in C. japonicum.

In Europe, silymarin, which is synthesized in Silybum marianum, belongs to the same family as $C$. japonicum and is prescribed for the treatment of chronic liver disease and the prevention of recurrent hepatitis $\mathrm{C}$ in liver transplant recipients [3, 4]. Silymarin is the key component of $S$. marianum and includes flavonolignans (silibinin, isosilibinin, silychristin, isosilychristin, and silydianin) and a flavonoid (taxifolin). Studies suggest that silymarin exerts a protective effect on the liver in alcoholic-induced liver disease, non-alcoholic fatty liver disease and carbon tetrachlorideinduced oxidative liver $[1,5]$. This major bioactivity has also been reported in C. japonicum DC, which has properties 
similar to those of S. marianum [6]. C. japonicum var. spinossimum is often compared to the basic species thistle (C. japonicum Fisch ex DC) [7]. Silymarin is synthesized through the phenylpropanoid pathway and transforms phenylalanine into 4-coumaroyl-CoA, which acts via both the flavonoid and monolignol pathways [8-10].

Significant progress has been achieved in the field of next generation sequencing, which is driven by genomic/ transcriptomic-based inquiries in biology. High-quality sequences are generated in a high-throughput manner at a low cost and with little labour. The Illumina platform is a highly utilized platform employed for transcriptome analyses of various model and non-model organisms and medicinal plants due to its potential for a high sequence yield [11-13]. The RNA sequencing (RNA-Seq) method is superior to whole genome sequencing because RNA-Seq only studies the transcribed regions and provides a comprehensive and integrated view of the transcriptome with the precise locations of the transcriptome boundaries [14]. RNA-Seq is currently a very popular method used to examine both coding and non-coding gene annotations. Remarkable progress has been achieved in the exploration of medicinal plants at the genomic and transcriptomic levels, and the ultimate goal is to identify genes that are involved in biologically active phytocompounds and related pathways [15-18].

In this study, we established transcript databases for $C$. japonicum var. spinossimum and provided additional genetic information for further genome-wide research and analyses. We also aimed to investigate the transcripts that contribute to the production of silymarin in this herb.

\section{Methods}

\section{Plant material, RNA extraction, and library preparation}

C. japonicum was obtained from the National Institute of Biological Resources, Korea. The flower, leaf, and root tissues were immediately dissected and grinded in liquid nitrogen for the RNA extraction. The total RNA was extracted using Hybrid-R (Geneall, PN: 3033522) according to the manufacturer's instructions. The total RNA was further treated with RNase-free DNase I (TaKaRa, Tokyo, Japan) and purified on an RNA-purification column (Qiagen, Valencia, CA, USA) to eliminate possible gnomic contamination. The RNA quality was evaluated using an Agilent 2100 Bioanalyzer (Agilent Technologies, Santa Clara, CA, USA), and RNA samples with RNA integrity number values above eight were used for the subsequent cDNA synthesis. The DNA was sheared with an average of $500 \mathrm{bp}$ fragment sizes. The TruSeq Library Preparation Kit (Illumina Inc., San
Diego, CA, USA) was used to construct the DNA library according to the manufacturer's protocol. The DNA libraries were sequenced with 150-bp paired-end sequencing using an Illumina Hiseq2500. The quality of the constructed libraries was confirmed by a LabChip GX system (PerkinElmer, Waltham, MA, USA).

\section{De novo assembly, functional annotation, Gene Ontology, and Kyoto Encyclopedia of Genes and Genomes pathway analysis}

The raw data were quality-filtered using trimmomatic with the following options: minimum quality of base (3); sliding window (4); average quality phred score (20); and minimum read size (50 bp). The primer and adapter sequences incorporated during cDNA synthesis were removed. The de novo assembly of the reads was performed using Trinity assembler with the default options to form the contigs. Based on the final transcriptome isoform sequences, the candidate-coding regions were identified using TransDecoder software (The Broad Institute, Cambridge, MA, USA). A BLAST analysis was carried out using these candidate-coding regions against the UniProt and NCBI non-redundant (nr) protein databases to determine the sequence similarity with genes from other species at an E-value cut-off of $1 \times 10^{-6}$. The functional categories of these sequences were matched to the Gene Ontology (GO) and Kyoto Encyclopedia of Genes and Genomes (KEGG) algorithms using blast. The GO analysis, including the biological function, cell component and molecular function categories, and the KEGG pathway analysis were performed using the GOstats program (Roswell Park Center Institute, Buffalo, NY, USA) as implemented in the sequence annotation tool Blast2GO (BioBam Bioinformatics SL, Valencia, Spain). The gene annotation and GO analysis were performed at NICEM, Seoul National University (Seoul, Korea). The assembled data were arranged according to the read length, GenBank number, E-value and species, and the specific composition of the GO terms was calculated and presented in a bar chart according to the percentage.

\section{Differential gene expression analysis}

The filtered raw reads were mapped to the C. japonicum contigs that were determined by the de novo assembly and annotation using HISAT2. The expression values in RNA-Seq were calculated based on the read count, and the expression levels were analysed using the counts (number of fragments) and FPKM (Fragments per Kilobase of transcript per Million fragments mapped reads). The differentially expressed gene (DEG) analysis among the leaf, flower and root tissues was carried out using the edgeR package [18]. The significantly DEGs were screened at a threshold false 
discovery rate $<0.05$ and an absolute log2-fold change value $>$ 1. Subsequently, GO functional enrichment and KEGG pathway analyses of the DEGs were performed.

\section{Results}

\section{De novo assembly}

To study the transcriptome of $C$. japonicum, three tissues, viz., flowers, leaves, and roots, were extracted, and the RNA was isolated. The total RNA from each sample was used for the mRNA preparation, fragmentation, cDNA synthesis and library preparation. De novo assembly was selected since a reference genome for $C$. japonicum is lacking. Each library was sequenced using the Illumina Hiseq2500 platform. The platform generated 74,566,546 raw reads for $C$. japonicum for the whole transcriptome, which accounted for $18 \mathrm{Gbases}$ of sequence data (Supplementary Table 1). These data were further subjected to a quality check and adaptor trimming (using the trimmomatic 0.30 tool), generating 67,153,874 high-quality paired end reads (Tables 1 and 2). Then, an error correction of the data was performed, and 66,078,302 reads were obtained. The paired end sequencing yielded on average a $64 \times$ sequencing depth. The mean Phred value was above 20 , and the short reads ( $>50$ ) were removed. The whole transcriptome was assembled using the Trinity program, resulting in 51,133 unigenes, accounting for 37 Mbases, with a mean size of 648.36 bp. The length distribution of all unigenes is shown in Fig. 1. The GC content was distributed within $42 \%-45 \%$. The minimum size of the transcripts was $147 \mathrm{bp}$, whereas the maximum size was 15,402 bp (Table 1 ).

Table 1. The total transcripts and length by de novo assembly of RNA sequence data in Cirsium japonicum

\begin{tabular}{lr}
\hline Assembly data & \multicolumn{1}{c}{ No. } \\
\hline Transcripts & 51,133 \\
Total (bp) & $37,533,942$ \\
Mean size (bp) & 648 \\
Maximum size & 15,402 \\
\hline
\end{tabular}

\section{Functional annotation and classification}

The transcripts derived from $C$. japonicum were subjected to annotation using the BLASTX program based on a homology search against the NCBI non-redundant (nr) protein database. The highest homologous subject in each contig was selected for gene annotation. The identified contigs were contigs that hit to variable species with a high similarity homologous BLAST search. In total, 33,525 (65\%) unigenes were annotated using the BLASTX program. The similarity distribution demonstrated that C. japonicum was highly similar to Cynara cardunculus var. scolymus (72\%) (Fig. 2). GO broadly categorizes genes into one of three classes (biological process, cellular component, and molecular function) to describe their involvement in plant biological phenomena. The transcript functions were predicted with the help of the GO terms using Blast2GO based on their similarities to transcripts available in the $\mathrm{nr}$ database. All assembled transcripts were surveyed at an E-value of $1 \times 10^{-6}$. The numbers of genes assigned to the biological process, molecular function, and cellular component categories were 9,336, 2,371, and 1,163, respectively (Fig. 3). Among the biological processes transcripts, the highest number of transcripts was assigned to cellular processes $(13,538)$,

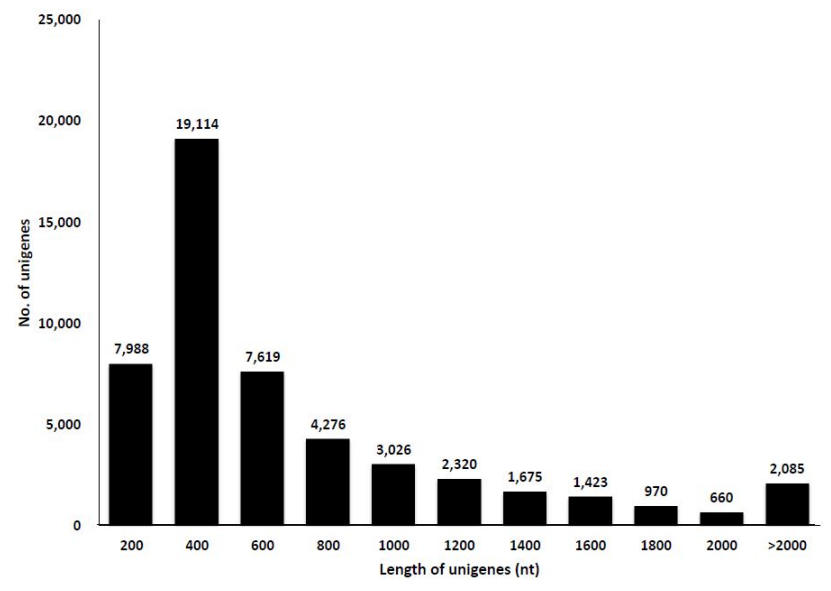

Fig. 1. Distribution of the length of the unigenes obtained by the de novo assembly of the transcriptome sequencing in Cirsium japonicum.

Table 2. The mapped reads to the transcripts using trimmed RNA sequence data

\begin{tabular}{lccccc}
\hline & \multicolumn{6}{c}{ Mapping reads to transcripts for DEG analysis } \\
\cline { 2 - 6 } & Trimmed reads & Mapped reads, $\mathbf{n}(\%)$ & No. of mapped transcripts & GC (\%) & Q30 (\%) \\
\hline Flower & $20,728,854$ & $9,751,931(47.05)$ & 24,663 & 44.88 & 96.29 \\
Leaf & $30,141,768$ & $16,009,899(53.12)$ & 34,031 & 42.9 & 96.46 \\
Root & $16,283,252$ & $8,121,204(49.87)$ & 19,545 & 44.88 & 96.34 \\
\hline
\end{tabular}


followed by metabolic processes (12,322) and single organism processes $(11,785)$. Several other processes, including biological regulation and response to stimulus, also comprised a significant number of transcripts (Fig, 3, biological process). The most prominent molecular functions included binding activity $(12,037)$ and catalytic activity $(10,141)$ (Fig. 3 , molecular function). In the cellular component analysis, most transcripts were assigned to cell $(12,808)$, cell part $(12,251)$, organelle $(8,806)$, and membrane $(8,272)$. Macromolecular complex $(5,632)$, membrane part $(5,265)$, and

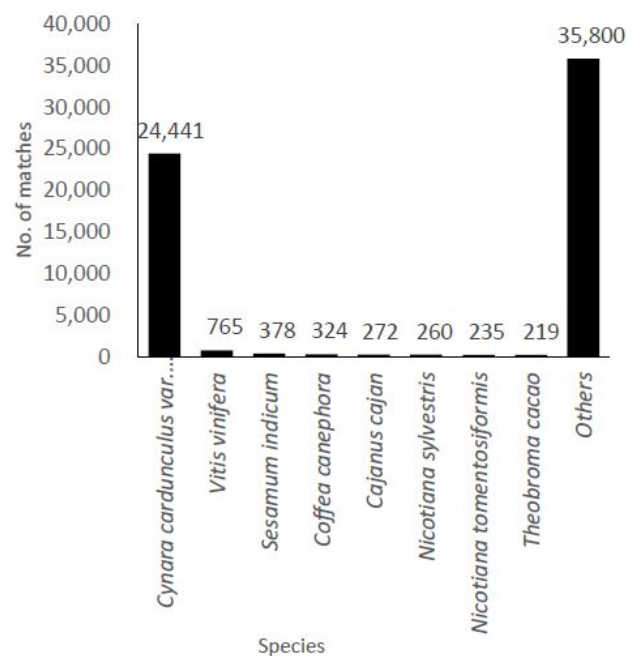

Fig. 2. Distribution of the species hits based on the gene annotation of the transcriptome by the BlastX search in Cirsium japonicum var. spinossimum. organelle part $(4,717)$ also had a substantial number of transcripts (Fig. 3, cellular component).

\section{Functional classification of KEGG pathways}

For the KEGG analysis, 17,583 annotated transcripts were mapped to identify the active pathways in C. japonicum at a cut-off E-value $<0.00001$. Of these transcripts, 9,418 transcripts had significant matches in the database and were assigned to 137 KEGG pathways. Metabolism had the most unigenes; amino acid metabolism had the highest number of annotated unigenes, followed by carbohydrate metabolism, xenobiotic biodegradation and metabolism, lipid metabolism, biosynthesis of antibiotics, etc. (Table 3). These annotations provide insight into the transcriptome by enhancing our understanding of the specific functions and pathways in $C$. japonicum.

\section{Screening and identification of DEGs}

To identify the differentially ex pressed genes, the transcriptome data from C. japonicum leaf, root and flower tissues were analysed using the EdgeR package in $\mathrm{R}$ software [19]. The DEGs were visualized as an MA plot (log ratio vs. abundance plot) of flower vs. leaf, flower vs. root and leaf vs. root (Supplementary Fig. 1). The red dots represent transcripts with positive and negative log2fold change values, indicating the up-regulation and down-regulation of the DEGs in each comparison. Using these criteria, most transcripts were mapped in the leaf tissue $(34,031)$, followed by the flower $(24,663)$ and root $(19,545)$ tissues (Table 2$)$. The expression

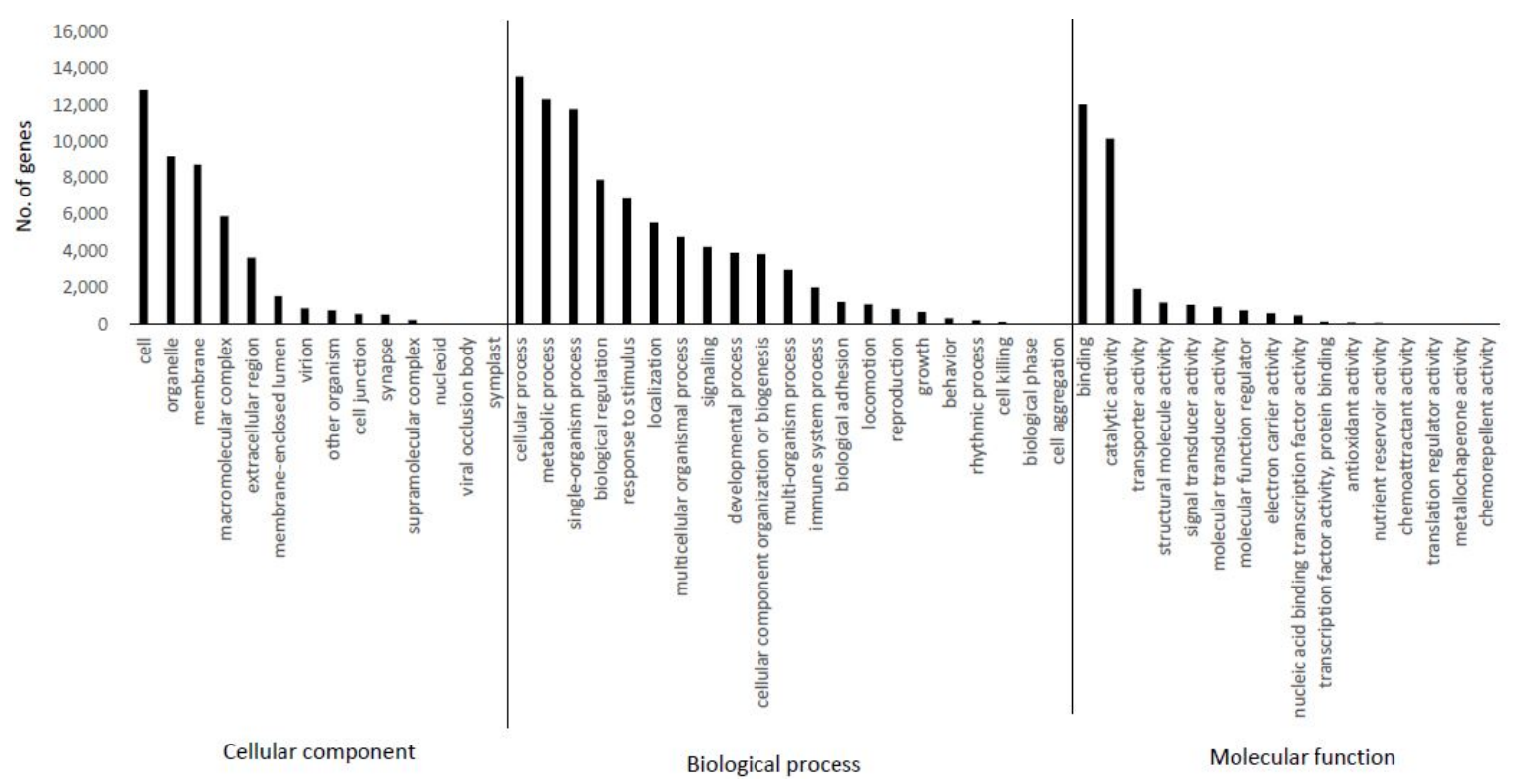

Fig. 3. Gene Ontology (GO) annotations of non-redundant consensus sequences. The best hits were aligned to the GO database, and the most consensus sequences were grouped into three major functional categories, namely, biological process, molecular function, and cellular component. 
Table 3. KEGG analysis pathway distribution among the transcriptome of Cirsium japonicum var. spinossimum

\begin{tabular}{|c|c|c|c|}
\hline $\begin{array}{l}\text { KEGG pathways } \\
\text { classification }\end{array}$ & Sub classification & $\begin{array}{l}\text { Count of } \\
\text { pathway }\end{array}$ & $\begin{array}{c}\text { Sum of unigenes } \\
\text { in pathway }\end{array}$ \\
\hline \multirow[t]{12}{*}{ Metabolism } & Amino acid metabolism & 14 & 1597 \\
\hline & Carbohydrate metabolism & 15 & 1354 \\
\hline & Xenobiotics biodegradation and metabolism & 18 & 1312 \\
\hline & Lipid metabolism & 15 & 1258 \\
\hline & Biosynthesis of antibiotics & 1 & 957 \\
\hline & Energy metabolism & 7 & 876 \\
\hline & Nucleotide metabolism & 2 & 519 \\
\hline & Metabolism of cofactors and vitamins & 12 & 509 \\
\hline & Biosynthesis of other secondary metabolites & 17 & 316 \\
\hline & Metabolism of other amino acids & 9 & 230 \\
\hline & Glycan biosynthesis and metabolism & 11 & 185 \\
\hline & Metabolism of terpenoids and polyketides & 9 & 62 \\
\hline Environmental information processing & Signal transduction & 3 & 117 \\
\hline Organismal systems & Immune system & 2 & 66 \\
\hline Genetic information processing & Translation & 1 & 44 \\
\hline Human disease & Drug resistance: antimicrobial & 1 & 16 \\
\hline Grand total & & 137 & 9,418 \\
\hline
\end{tabular}

KEGG, Kyoto Encyclopedia of Genes and Genomes.

Table 4. Number of expressed read counts from 51,133 transcripts in Cirsium japonicum

\begin{tabular}{lcc}
\hline & Expressed transcripts & Percentage \\
\hline Flower & 37,665 & 73.66 \\
Leaf & 46,991 & 91.90 \\
Root & 32,257 & 63.08 \\
\hline
\end{tabular}

values corresponding to specific genes were arranged in a matrix form, and two tables of the counts and FPKM were generated to represent the genes (data not shown). The count matrix represented the number of reads mapped to the reference, and the FPKM matrix represented the normalized value using the FPKM-based count value. In total, 51,133 expressed reference sequences were obtained. Overall, the leaf (Cjapleaf) samples had the most expressed transcripts $(46,991,91.90 \%)$, followed by the flower (Cjapflower) $(37,665,73.66 \%)$ and root (Cjaproot) $(32,257,63.08 \%)$, as shown in Table 4. Based on the expression differences shown in the Venn diagram in Fig. 4, GO and KEGG analyses were performed again based on the $\mathrm{nr}$ database annotations. The unigenes were assigned to $61 \mathrm{GO}$ categories as follows: Cjapleaf vs. Cjaproot (29,491 up-regulated and 27,898 down-regulated unigenes) (Supplementary Fig. 2); Cjaproot vs. Cjapflower (16,365 up-regulated and 32,596 downregulated unigenes) (Supplementary Fig. 3) and Cjapflower vs Cjapleaf (19,207 up-regulated and 33,472 downregulated unigenes) (Supplementary Fig. 4).

In the KEGG analysis, 2,615 unigenes were annotated in

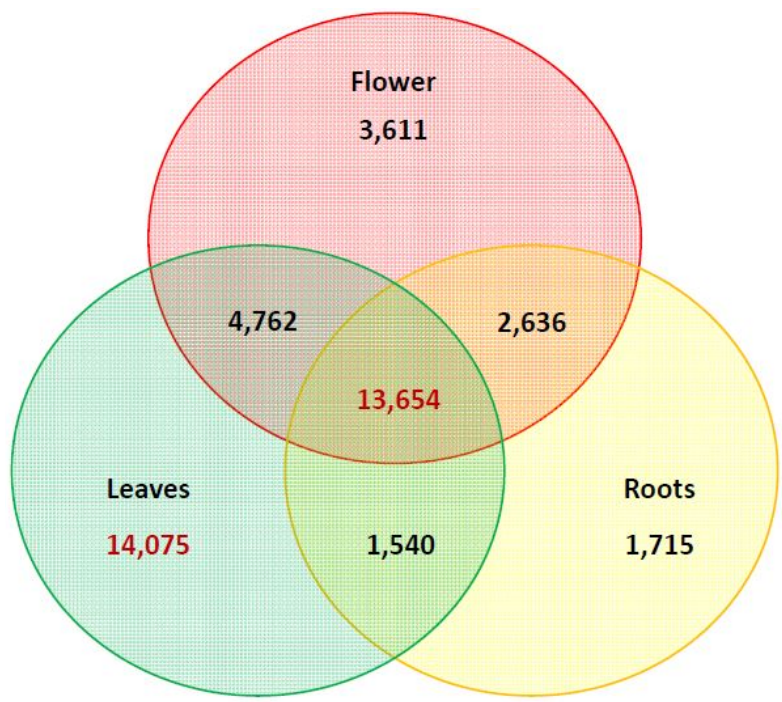

Fig. 4. Venn diagram representing the significantly differentially expressed transcripts among flower, leaf, and root tissues from Cirsium japonicum. The number includes both the up- and down-regulated genes. Genes that are differentially expressed in more than one comparison are depicted in the overlapping area.

the Cjapleaf vs. Cjaproot comparison; of these unigenes, 2,115 up-regulated unigenes were assigned to 109 pathways, and 500 down-regulated unigenes were assigned to 113 pathways. In the comparison Cjaproot vs. Cjapflower, 877 unigenes were annotated in the KEGG classification; of these unigenes, 348 up-regulated unigenes were assigned to 103 pathways, and 529 down-regulated unigenes were assigned to 116 pathways. In the comparison Cjapflower vs. Cjapleaf, 
934 unigenes were annotated; of these unigenes, 383 up-regulated unigenes were assigned to 97 pathways, and 551 down-regulated unigenes were assigned to 114 pathways. The differences in the expression levels were two-fold or greater, indicating a significant difference between the compared samples. The comparison of the up-regulated and down-regulated transcripts was performed by comparison among the tissues.

\section{Identification of putative unigenes involved in silymarin biosynthesis}

Silybin A and B, iso-silybins A and B, silychristin A, isosilychristin, and silydianin are all collectively called silymarins. Silymarins are the major flavolignans produced in C. japonicum. Taxifolin and coniferyl alcohol are the two major precursors responsible for the production of silymarin [20] and are produced by the flavonoid and phenylpropanoid pathways, respectively, as shown in Fig. 5 (the highlighted boxes represent the enzymes annotated in our data). The unigenes identified for the silymarin production in $C$. japonicum are listed in Supplementary Table 2. Most enzymes responsible for the production of silymarin were found in all three tissues (flower, leaf, and root) of C. japonicum. Subsequently, silymarin formation occurs via the oxidative

Coniferyl alcohol pathway

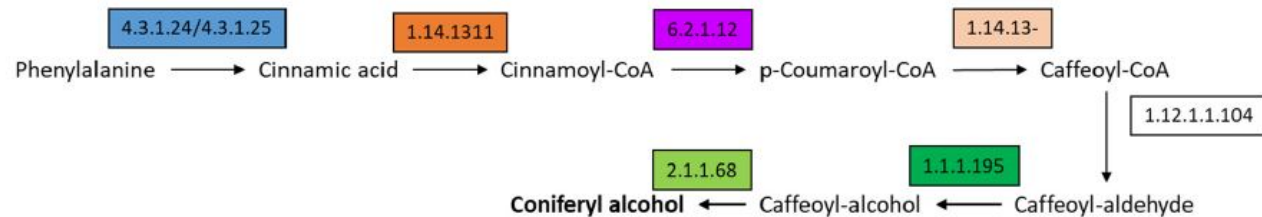

Taxifolin biosynthesis pathway

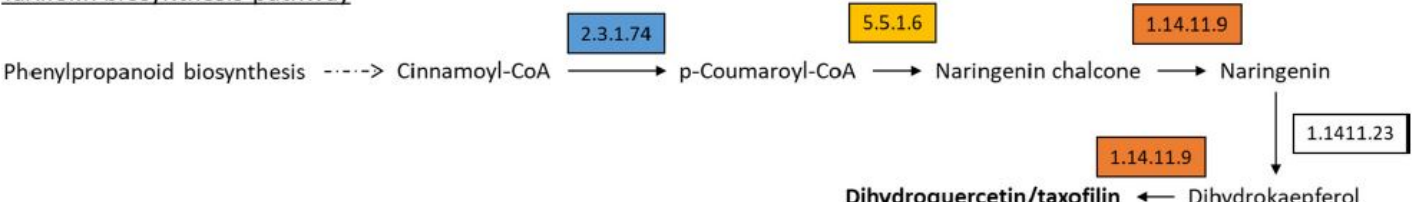

Fig. 5. Silymarin is produced via the oxidative coupling of two major precursors, namely, taxifolin and coniferyl alcohol. The figure represents the formation of coniferyl alcohol and taxifolin via the phenylpropanoid and flavonoid pathways, respectively. The number in the boxes represents the enzyme as code per the Kyoto Encyclopedia of Genes and Genomes (KEGG) classification, and the details are provided in Supplementary Table 2. The coloured boxes represent the enzymes that were found in our transcriptome data.

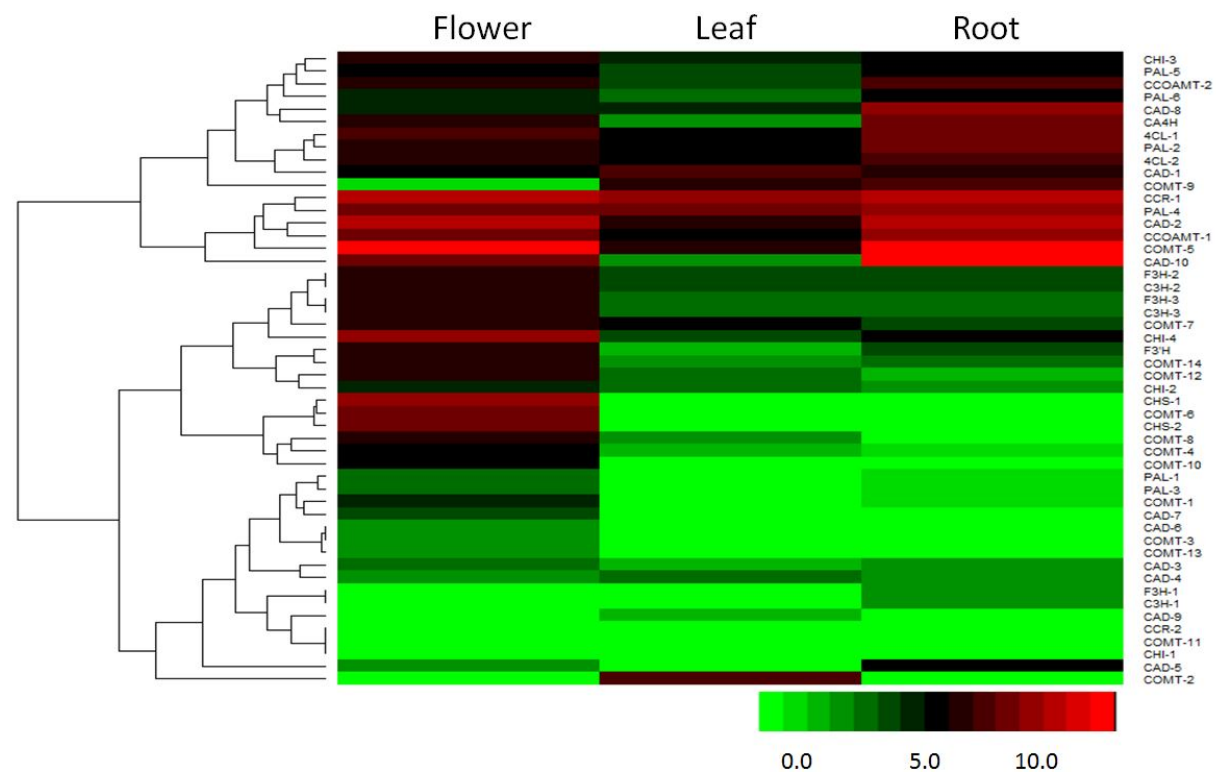

Fig. 6. Heat map of the differently expressed genes involved in silymarin biosynthesis. Red and green colours indicate up-and down-regulated gene expression, respectively. 
radicalization of taxifolin and coniferyl alcohol, followed by a combinational radical coupling [21]. Genes corresponding to the silymarin biosynthetic pathway (flavonoid and phenylpropanoid pathway-related genes) were selected from the C. japonicum transcripts, and the expression patterns were compared among the flower, leaf and root tissues (Supplementary Table 2). The organ-specific gene expression patterns in C. japonicum were confirmed by the heat map technique (Fig. 6). The maximum number of genes was found to be downregulated in leaf tissues.

\section{Discussion}

Cirsium japonicum var. spinossimum is a wild perennial herb that grows in the mountains and fields of Jeju Island, South Korea, and is also found in Japan. This species is often compared to a similar herb called C. japonicum DC [7]. In Korea, this herb is known for its medicinal properties, including its antihaemorrhagic, antihypertensive, antihepatitic, and uretic medicinal properties [2]. Since the advent of next generation technology, this research tool has become important for the generation of vast information regarding genomic and transcriptomic data. These data eventually help infer various basic biological, molecular and cellular processes, especially in non-model organisms and non-sequenced genomes [22-24]. Thus far, no studies have performed a transcriptome analysis of this herb. We characterized the transcriptome of $C$. japonicum var. spinossimum using an Illumina Hiseq2500. This study represents the first report of the whole transcriptome and differential gene expression among the flower, leaf, and root tissues in C. japonicum var. spinossimum and provides insight into fundamental molecular data.

We obtained 66,078,874 reads after trimming and performing error corrections, and these reads assembled into 51,133 unigenes with an average length of $648 \mathrm{bp}$ (Table $1)$. The number of mapped transcripts in each tissue was $24,663,34,031$, and 19,545 in the flower, leaf, and root tissues, respectively (Table 2 ). We obtained unigenes that ranged from 200 to 15,402 bp (Fig. 1), which is comparable to similar RNA-Seq reports [25-27]. This number also represents the high quality of our generated data. Although prior genome information for $C$. japonicum is lacking, the BLASTX program successfully annotated 33,525 (65.59\%) transcripts. The highest similarity obtained with Cynara cardunculus var. scolymus, is a medicinal plant belonging to same family (Asteraceae) [28, 29]. Thus, the C. japonicum transcriptome can prove useful for functional gene studies or molecular biology studies investigating $C$. japonicum. Of the 17,583 mapped transcripts, the KEGG classification annotated 9,418 (53.56\%) transcripts into several pathways
(Table 3), but no functional annotation was found for the remaining $46.44 \%$ of the assembled unigenes. This might be due to one of the following reasons: either the unigenes matched proteins with unknown functions or they did not have any homologous sequence matches in the database. Thus, these unknown unigenes may be highly important for further research since they may be considered novel transcripts.

The DEG patterns were investigated to further profile the global gene expression differences among the leaf, flower and root in C. japonicum. The most abundant genes were expressed in the leaves, followed by the flowers and roots (Fig. 4). We obtained 26,289 genes that were expressed in all three tissues, including 14,075 genes that were specifically expressed in the leaf tissues, 3,611 genes that were specifically expressed in the flower tissues and 1,715 genes that were specifically expressed in the root tissues. The molecular function category showed that more than $40 \%$ of the unigenes were expressed in all three tissues (Supplementary Figs. 2-4). The major categories in which differential gene expression was observed were biological process and cellular components.

Silymarin is a main component in $C$. japonicum that contributes to its medicinal value. Therefore, we aimed to expand our knowledge by identifying putative unigenes that contribute to the product. Silymarin is reportedly produced via the oxidative coupling of two major precursors, namely, taxifolin and coniferyl alcohol [21]. Most major enzymes responsible to produce both taxifolin and coniferyl alcohol were present in our transcriptome data (Supplementary Table 2), which is consistent with other medicinal plants observed in the same family $[6,20]$. In the C. japonicum transcriptome assembly, we identified silymarin biosynthesisrelated unigenes (Fig. 5), and their differential expression was assessed (Fig. 6). Compared to their expression in the flower and root tissues, these genes were highly expressed in the leaf tissues. Silymarin biosynthesis has been reported to be lacking in the flower of S. marianum [6].

In addition to these unigenes, unigenes encoding enzymes crucial for the biosynthesis of antibiotics and the biosynthesis of secondary metabolites were identified. Medicinal plants represent a rich source of secondary metabolites [30, 31], and their data could be useful for molecular biology research and the mass production of significant metabolites.

This report represents the first de novo assembly of the transcriptome of $C$. japonicum var. spinossimum obtained from the Korean Peninsula. This study provides resources for comparative transcriptomics, especially in the field of the biochemical and molecular biosynthesis pathways of silymarin. In total, 51,133 unigenes were obtained with a mean length of 648.36 bp. In total, 33,525 annotated 
sequences were assigned to $64 \mathrm{GO}$ functional groups, and 10,535 unigenes were assigned to 107 KEGG pathways. The DEG analysis revealed that the highest numbers of genes were expressed in the leaf tissues. Candidate genes that might be involved in silymarin biosynthesis may help further functional genomic and transcriptomic analyses in $C$. japonicum. Putative unigenes that facilitate the production of taxifolin and coniferyl alcohol, which are the major precursors in silymarin biosynthesis, were identified. This work will contribute to the comprehensive knowledge of new traditional medicinal plants for growers and consumers and provides additional characteristics and information regarding the pharmaceutical benefits associated with silymarin biosynthesis.

ORCID: Neha Samir Roy: https://orcid.org/0000-00024529-4861; Jung-A Kim: https://orcid.org/0000-0002-01365410; Ah-Young Choi: https://orcid.org/0000-0002-20136533; Yong-Wook Ban: https://orcid.org/0000-0002-19120374; Nam-Il Park: https://orcid.org/0000-0001-6725-0758; Kyong-Cheul Park: https://orcid.org/0000-0002-3737-815X; Hee-sun Yang: https://orcid.org/0000-0002-3289-6345; IkYoung Choi: https://orcid.org/0000-0003-4168-0471; Soo nok Kim: https://orcid.org/0000-0003-1654-3643

\section{Authors' contribution}

\author{
Conceptualization: SK, IYC \\ Data curation: NSR, AYC, YWB, KCP \\ Formal analysis: NSR, YWB \\ Funding acquisition: SK, HSY \\ Methodology: IYC, NSR, NIP, JAK \\ Writing - original draft: NSR, YWB \\ Writing - review \& editing: NSR, YWB, SK, IYC
}

\section{Conflicts of Interest}

No potential conflicts of interest relevant to this article was reported.

\section{Acknowledgments}

This work was supported by the grants from 2017 Research Grant, Kangwon National University and the National Institute of Biological resources (NIBR), funded by the Minister of Environment (MOE) of the Republic of Korea (NIBR201626103, NIBR201830101).

\section{Supplementary material}

Supplementary data including two tables and four figures can be found with this article online at https://doi.org/10.5808/ GI.2018.16.4.e34.

\section{References}

1. Ge H, Turhong M, Abudkrem M, Tang Y. Fingerprint analysis of Cirsium japonicum DC. using high performance liquid chromatography. J Pharm Anal 2013;3:278-284

2. Liu S, Luo X, Li D, Zhang J, Qiu D, Liu W, et al. Tumor inhibition and improved immunity in mice treated with flavone from Cirsium japonicum DC. Int Immunopharmacol 2006;6:13871393.

3. Federico A, Dallio M, Loguercio C. Silymarin/silybin and chronic liver disease: a marriage of many years. Molecules 2017;22:E191.

4. Loguercio C, Festi D. Silybin and the liver: from basic research to clinical practice. World J Gastroenterol 2011;17:2288-2301.

5. Kim NC, Graf TN, Sparacino CM, Wani MC, Wall ME. Complete isolation and characterization of silybins and isosilybins from milk thistle (Silybum marianum). Org Biomol Chem 2003;1:1684-1689.

6. Ma Q, Wang LH, Jiang JG. Hepatoprotective effect of flavonoids from Cirsium japonicum DC on hepatotoxicity in comparison with silymarin. Food Funct 2016;7:2179-2184.

7. WoRMS. Cirsium japonicum var. spinosissimum Kitam. WoRMS, 2008. Accessed 2018 Nov 1. Available from: http://www. marinespecies.org/aphia.php? $\mathrm{p}=$ taxdetails\&id $=1157330$.

8. Boerjan W, Ralph J, Baucher M. Lignin biosynthesis. Annu Rev Plant Biol 2003;54:519-546.

9. Morazzoni P, Bombardelli E. Silybum marianum (Carduus marianus). Fitoterapia 1995;66:3-42.

10. Petrussa E, Braidot E, Zancani M, Peresson C, Bertolini A, Patui $S$, et al. Plant flavonoids: biosynthesis, transport and involvement in stress responses. Int J Mol Sci 2013;14: 14950-14973.

11. Nakasugi K, Crowhurst RN, Bally J, Wood CC, Hellens RP, Waterhouse PM. De novo transcriptome sequence assembly and analysis of RNA silencing genes of Nicotiana benthamiana. PLoS One 2013;8:e59534.

12. Lehnert EM, Walbot V. Sequencing and de novo assembly of a Dahlia hybrid cultivar transcriptome. Front Plant Sci 2014;5:340.

13. Rastogi S, Meena S, Bhattacharya A, Ghosh S, Shukla RK, Sangwan NS, et al. De novo sequencing and comparative analysis of holy and sweet basil transcriptomes. BMC Genomics 2014; 15:588.

14. Wilhelm BT, Marguerat S, Goodhead I, Bähler J. Defining transcribed regions using RNA-seq. Nat Protoc 2010;5:255-266.

15. Martínez-García PJ, Crepeau MW, Puiu D, Gonzalez-Ibeas D, Whalen J, Stevens KA, et al. The walnut (Juglans regia) genome sequence reveals diversity in genes coding for the biosynthesis of non-structural polyphenols. Plant $J$ 2016;87:507-532.

16. Liu MH, Yang BR, Cheung WF, Yang KY, Zhou HF, Kwok JS, et al. Transcriptome analysis of leaves, roots and flowers of Panax notoginseng identifies genes involved in ginsenoside and alkaloid biosynthesis. BMC Genomics 2015;16:265.

17. Lulin $H$, Xiao $Y$, Pei $S$, Wen $T$, Shangqin $H$. The first Illumina-based de novo transcriptome sequencing and analysis 
of safflower flowers. PLoS One 2012;7:e38653.

18. Upadhyay AK, Chacko AR, Gandhimathi A, Ghosh P, Harini K, Joseph AP, et al. Genome sequencing of herb Tulsi (Ocimum tenuiflorum) unravels key genes behind its strong medicinal properties. BMC Plant Biol 2015;15:212.

19. Robinson MD, McCarthy DJ, Smyth GK. edgeR: a bioconductor package for differential expression analysis of digital gene expression data. Bioinformatics 2010;26:139-140.

20. AbouZid SF, Ahmed HS, Moawad AS, Owis AI, Chen SN, Nachtergael A, et al. Chemotaxonomic and biosynthetic relationships between flavonolignans produced by Silybum marianum populations. Fitoterapia 2017;119:175-184.

21. Lv Y, Gao S, Xu S, Du G, Zhou J, Chen J. Spatial organization of silybin biosynthesis in milk thistle [Silybum marianum (L.) Gaertn]. Plant J 2017;92:995-1004.

22. Mudalkar S, Golla R, Ghatty S, Reddy AR. De novo transcriptome analysis of an imminent biofuel crop, Camelina sativa L. using Illumina GAIIX sequencing platform and identification of SSR markers. Plant Mol Biol 2014;84:159-171.

23. Yang Y, Xu M, Luo Q, Wang J, Li H. De novo transcriptome analysis of Liriodendron chinense petals and leaves by Illumina sequencing. Gene 2014;534:155-162.

24. Zhu L, Zhang Y, Guo W, Xu XJ, Wang Q. De novo assembly and characterization of Sophora japonica transcriptome using RNA-seq. Biomed Res Int 2014;2014:750961.
25. Zhang X, Allan AC, Li C, Wang Y, Yao Q. De novo assembly and characterization of the transcriptome of the Chinese medicinal herb, Gentiana rigescens. Int J Mol Sci 2015;16:11550-11573.

26. Zhang X, Liu T, Duan M, Song J, Li X. De novo transcriptome analysis of Sinapis alba in revealing the glucosinolate and phytochelatin pathways. Front Plant Sci 2016;7:259.

27. Sun H, Li F, Xu Z, Sun M, Cong H, Qiao F, et al. De novo leaf and root transcriptome analysis to identify putative genes involved in triterpenoid saponins biosynthesis in Hedera helix L. PLoS One 2017;12:e182243.

28. Acquadro A, Barchi L, Portis E, Mangino G, Valentino D, Mauromicale G, et al. Genome reconstruction in Cynara cardunculus taxa gains access to chromosome-scale DNA variation. Sci Rep 2017;7:5617.

29. Scaglione D, Lanteri S, Acquadro A, Lai Z, Knapp SJ, Rieseberg $\mathrm{L}$, et al. Large-scale transcriptome characterization and mass discovery of SNPs in globe artichoke and its related taxa. Plant Biotechnol J 2012;10:956-969.

30. Hartmann T. Plant-derived secondary metabolites as defensive chemicals in herbivorous insects: a case study in chemical ecology. Planta 2004;219:1-4.

31. Goyal S, Lambert C, Cluzet S, Merillon JM, Ramawat KG. Secondary metabolites and plant defence. In: Plant Defence: Biological Control (Merillon JM, Ramawat KG, eds.). Dordrecht: Springer, 2012. pp. 109-138. 


\section{SUPPLEMENTARY INFORMATION}

\section{RNA-Seq De Novo Assembly and Differential Transcriptome Analysis of} Korean Medicinal Herb Cirsium japonicum var. spinossimum

\section{Neha Samir Roy ${ }^{1,2}$, Jung-A Kim ${ }^{3}$, Ah-Young Choi ${ }^{4}$, Yong-Wook Ban ${ }^{5}$, Nam-Il Park ${ }^{6}$, Kyong-Cheul Park', Hee-sun Yang ${ }^{3}$, Ik-Young Choi ${ }^{1,2 *}$, Soonok Kim ${ }^{3 * *}$}

\footnotetext{
${ }^{1}$ Department of Agriculture and Life Industry, Kangwon National University, Chuncheon 24341, Korea, ${ }^{2}$ Agriculture and Life Sciences Research Institute, Kangwon National University, Chuncheon 24341, Korea, ${ }^{3}$ Biological Resources Assessment Division, National Institute of Biological Resources, Incheon 22689, Korea, ${ }^{4}$ DNACare Genomics Institute, Seongnam 13558, Korea, ${ }^{5}$ Department of Forest Environmental System, Kangwon National University, Chuncheon 24341, Korea, ${ }^{6}$ Department of Plant Science, Gangneung Wonju National University, Gangneung 25457, Korea
} 
Supplementary Table 1. Summary of data output from Illumina HiSeq2500 for Cirsium japonicum var. spinossimum

\begin{tabular}{|c|c|c|c|c|c|}
\hline & Sample: Cjap reference & Reads & Read bases & Average & Largest length \\
\hline \multirow{3}{*}{$\begin{array}{l}\text { Reference sequencing data } \\
\text { summary }\end{array}$} & Raw data & $74,566,546$ & $18,716,203,046$ & 251 & 251 \\
\hline & Trimmed data & $67,153,874$ & $12,346,643,676$ & 183.86 & 251 \\
\hline & Error correction data & $66,078,302$ & $11,841,793,951$ & 179.21 & 251 \\
\hline Reference assembly summary & $\begin{array}{l}\text { Trinity (RNA-seq } d e \\
\text { novo assembly) } \\
\text { Transdecoder }\end{array}$ & 51,133 & $37,533,942$ & 648.36 & 15,402 \\
\hline
\end{tabular}


Supplementary table 2. Log2Fold change values obtained for the enzymes involved in Coniferyl alcohol and Taxifolin pathways for Heat map Enzymes for Coniferyl alcohol pathway

1. phenylalanine ammonia-lyase [EC:4.3.1.24]_PAL

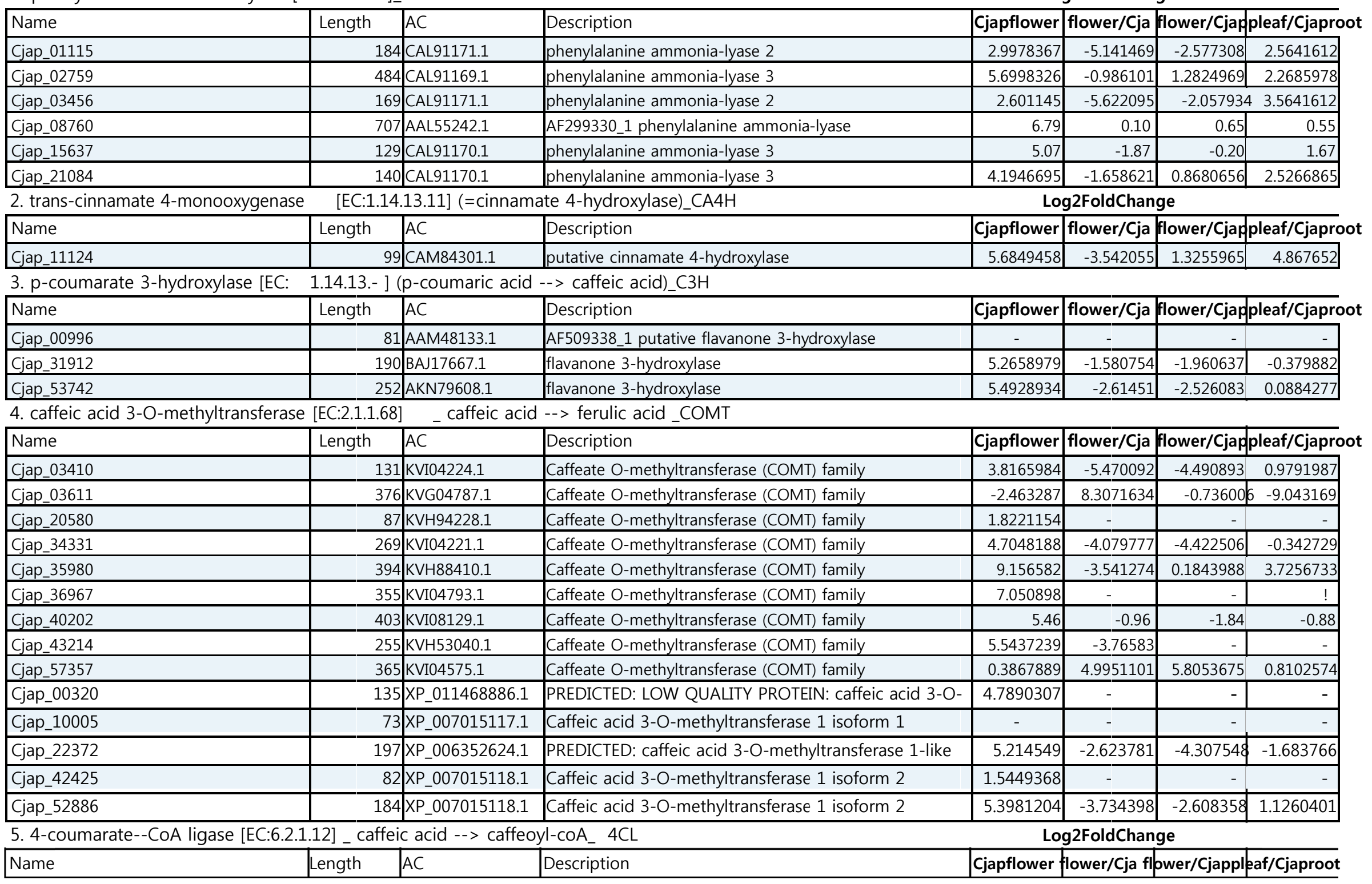

Log2FoldChange

Cjapflower flower/Cja flower/Cjappleaf/Cjaproo \begin{tabular}{|l|c|r|r|r|}
\hline Description & Cjapflower & flower/Cja & flower/Cjap pleaf/Cjaproot \\
\hline Af509338_1 putative flavanone 3-hydroxylase & - & - & - & - \\
\hline flavanone 3-hydroxylase & 5.2658979 & -1.580754 & -1.960637 & -0.379882 \\
\hline flavanone 3-hydroxylase & 5.4928934 & -2.61451 & -2.526083 & 0.0884277 \\
\hline
\end{tabular} --> ferulic acid_COMT 


\begin{tabular}{|c|c|c|c|c|c|c|}
\hline Cjap_07087 & \begin{tabular}{l|l|}
539 & AFL93685.1
\end{tabular} & 4-coumarate: coenzyme A ligase & 6.05 & -0.98 & 0.62 & 1.60 \\
\hline Cjap_27264 & 531|XP_009603383.1 & PREDICTED: 4-coumarate--CoA ligase-like 7 & 5.6059055 & -0.496624 & 0.4001129 & 0.8967365 \\
\hline
\end{tabular}

6. cinnamoyl-CoA reductase [EC:1.2.1.44] _ caffeoyl-coA --> caffeyl-aldehyde _ CCR

\begin{tabular}{|c|c|c|c|c|c|c|c|}
\hline Name & Length & $A C$ & Description & Cjapflower & flower/Cja f & flower/Cjap & pleaf/Cjaproc \\
\hline Cjap_19620 & 324 & XP_002275195.1 & PREDICTED: cinnamoyl-CoA reductase 1 & 8.0614507 & -0.606384 & -0.240069 & 0.3663147 \\
\hline Cjap_32839 & 344 & XP_002300619.2 & cinnamoyl-CoA reductase family protein & & & & \\
\hline
\end{tabular}

7. cinnamyl-alcohol dehydrogenase [EC:1.1.1.195]_caffeyl-aldehyde --> caffeyl alcohol_CAD

\begin{tabular}{|c|c|c|c|c|c|c|c|}
\hline Name & Length & AC & Description & Cjapflower $\mathrm{f}$ & ower/Cja & ower/Cjap & leaf/Cjapro \\
\hline Cjap_03029 & 360 & XP_012073738.1 & PREDICTED: probable cinnamyl alcohol dehydrogenase & 4.875403 & 1.221775 & 0.287078 & -0.9347 \\
\hline Cjap_04466 & 386 & BAN91671.1 & cinnamyl alcohol dehydrogenase 2 & 8.136022 & -2.45746 & -0.34242 & 2.115048 \\
\hline Cjap_04954 & 165 & BAN91672.1 & cinnamyl alcohol dehydrogenase 3 & 2.429251 & -1.16772 & -0.18852 & 0.979199 \\
\hline Cjap_20491 & 206 & BAN91672.1 & cinnamyl alcohol dehydrogenase 3 & 1.578558 & 0.787296 & 0.00096 & -0.78634 \\
\hline Cjap_26535 & 88 & BAN91672.1 & cinnamyl alcohol dehydrogenase 3 & 1.941329 & - & 2.98646 & 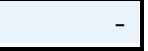 \\
\hline Cjap_27993 & 77 & BAN91670.1 & cinnamyl alcohol dehydrogenase 1 & 1.828347 & - & - & - \\
\hline Cjap_33336 & 89 & BAN91670.1 & cinnamyl alcohol dehydrogenase 1 & 3.142962 & - & - & - \\
\hline Cjap_41375 & 102 & BAN91672.1 & cinnamyl alcohol dehydrogenase 3 & 4.189993 & -0.26775 & 3.10102 & 3.368765 \\
\hline Cjap_50558 & 91 & BAN91672.1 & cinnamyl alcohol dehydrogenase 3 & - & - & - & - \\
\hline Cjap_51684 & 368 & BAN91670.1 & cinnamyl alcohol dehydrogenase 1 & 6.999154 & -5.01742 & 2.733929 & 7.751348 \\
\hline
\end{tabular}

8. caffeoyl-CoA O-

methyltransferase [EC: 2.1.1.104]

\begin{tabular}{|c|c|c|c|c|c|c|c|}
\hline Name & Length & $A C$ & Description & Cjapflower & flower/Cja & flower/Cjap & oleaf/Cjaproo \\
\hline Cjap_26162 & 247 & BAG71891.1 & caffeoyl CoA O-methyltransferase & 6.511625 & -1.566256 & 1.2530564 & 2.819312 \\
\hline Cjap_48779 & 172 & BAG71892.1 & caffeoyl CoA O-methyltransferase & 5.8153237 & -2.460632 & 0.2436025 & 2.7042341 \\
\hline
\end{tabular}

\section{Enzymes for Taxifolin pathway}

1. phenylalanine ammonia-lyase [EC:4.3.1.24]

\begin{tabular}{|l|r|l|}
\hline Name & Length & AC \\
\hline Cjap_01115 & 184 & CAL91171.1 \\
\hline Cjap_02759 & 484 & CAL91169.1 \\
\hline Cjap_03456 & 169 & CAL91171.1 \\
\hline Cjap_08760 & 707 & AAL55242.1 \\
\hline Cjap_15637 & 129 & CAL91170.1 \\
\hline Cjap_21084 & 140 & CAL91170.1 \\
\hline
\end{tabular}

Description

Log2FoldChange

2. trans-cinnamate 4-monooxygenase [EC:1.14.13.11] (=cinnamate 4-hydroxylase)

\begin{tabular}{|l|l|l|l}
\hline Name & Length & AC & Description \\
\hline
\end{tabular}

henylatanine ammonia-lyase 2

Cjapflower flower/Cja flower/Cjappleaf/Cjaproot

phenylalanine ammonia-lyase 3

phenylalanine ammonia-lyase 2

AF299330_1 phenylalanine ammonia-lyase

phenylalanine ammonia-lyase 3

\begin{tabular}{ll|lll|}
2.9978367 & -5.141469 & -2.577308 & 2.5641612 \\
\hline 5.6998326 & -0.986101 & 1.2824969 & 2.2685978
\end{tabular}

\begin{tabular}{|l|l|l|l|}
5.6998326 & -0.986101 & 1.2824969 & 2.2685978 \\
\hline
\end{tabular}

\begin{tabular}{|r|r|r|r|}
\hline 2.601145 & -5.622095 & -2.057934 & 3.5641612 \\
\hline
\end{tabular}

\begin{tabular}{|r|r|r|r|}
\hline 6.79 & 0.10 & 0.65 & 0.55 \\
\hline 5.07 & -1.87 & -0.20 & 1.67 \\
\hline
\end{tabular}

\begin{tabular}{r|r|rr|}
\hline 5.07 & -1.87 & -0.20 & 1.67 \\
\hline 4.1946695 & -1.658621 & 0.8680656 & 2.5266865 \\
\hline
\end{tabular}

Log2FoldChange

Cjapflower flower/Cja flower/Cjappleaf/Cjaproot 


\begin{tabular}{|c|c|c|c|c|c|c|c|c|}
\hline Cjap_11124 & & 99 & CAM84301.1 & putative cinnamate 4-hydroxylase & 5.6849458 & -3.542055 & 1.3255965 & 4.867652 \\
\hline \multicolumn{5}{|c|}{ 3. 4-coumarate--CoA ligase [EC:6.2.1.12] } & \multicolumn{3}{|c|}{ Log2FoldChange } & \\
\hline Name & Length & & $A C$ & Description & Cjapflower & flower/Cja f & flower/Cjapp & oleaf/Cjaproo \\
\hline Cjap_07087 & & 539 & AFL93685.1 & 4-coumarate: coenzyme A ligase & 6.05 & -0.98 & 0.62 & 1.60 \\
\hline Cjap_27264 & & 531 & XP_009603383.1 & PREDICTED: 4-coumarate--CoA ligase-like 7 & 5.6059055 & -0.496624 & 0.4001129 & 0.8967365 \\
\hline \multicolumn{5}{|c|}{ 4. chalcone synthase [EC:2.3.1.74] } & \multicolumn{3}{|c|}{ Log2FoldChange } & \\
\hline \multicolumn{3}{|l|}{ Name } & $A C$ & Description & Cjapflower & \multicolumn{3}{|c|}{\begin{tabular}{|l|l|l|} 
flower/Cja flower/Cjappleaf/Cjaproo \\
\end{tabular}} \\
\hline Cjap_00191 & & 405 & ABC71308.1 & chalcone synthase & 7.18 & -8.89 & - & - \\
\hline Cjap_17518 & & 401 & KVI02820.1 & Chalcone/stilbene synthase, active site-containing prote & 6.6437545 & -8.914877 & - & - \\
\hline \multicolumn{5}{|c|}{ 5. chalcone isomerase [EC:5.5.1.6] } & \multicolumn{3}{|c|}{ Log2FoldChange } & \\
\hline Name & Length & & $A C$ & Description & Cjapflower & \multicolumn{3}{|c|}{ flower/Cja flower/Cjappleaf/Cjaproc } \\
\hline Cjap_01214 & & 54 & KVI06946.1 & Chalcone isomerase & - & - & - & - \\
\hline Cjap_20743 & & 294 & KVI03948.1 & Chalcone isomerase, partial & 4.08 & -1.07 & -2.40 & -1.33 \\
\hline Cjap_21018 & & 410 & KVG40999.1 & Chalcone isomerase & 5.442806 & -1.26206 & -0.50458 & 0.757478 \\
\hline Cjap_22891 & & 243 & Q8LKP9.1 & CFI_SAUME RecName: Full=Chalcone--flavonone isome & 7.287225 & -3.65812 & $-2.3431 \beta$ & 1.314989 \\
\hline \multicolumn{5}{|c|}{ 6. flavanone 3-hydroxylase [EC:1.14.11.9] } & \multicolumn{3}{|c|}{ Log2FoldChange } & \\
\hline \multicolumn{3}{|l|}{ Name } & $A C$ & Description & Cjapflower & \multicolumn{3}{|c|}{ flower/Cja flower/Cjap/pleaf/Cjaproo } \\
\hline \multicolumn{2}{|l|}{ Cjap_00996 } & 81 & AAM48133.1 & AF509338_1 putative flavanone 3-hydroxylase & - & - & - & - \\
\hline \multicolumn{2}{|l|}{ Cjap_31912 } & 190 & BAJ17667.1 & flavanone 3-hydroxylase & 5.2658979 & -1.580754 & -1.960637 & -0.379882 \\
\hline Cjap_53742 & & 252 & AKN79608.1 & flavanone 3-hydroxylase & 5.4928934 & -2.61451 & -2.526083 & 0.0884277 \\
\hline \multicolumn{9}{|c|}{ 7. flavonoid 3',5'-hydroxylase [EC:1.14.13.88] } \\
\hline Name & Length & & $A C$ & Description & Cjapflower & \multicolumn{3}{|c|}{ flower/Cja flower/Cjap/pleaf/Cjaproo } \\
\hline Cjap_08727 & & 517 & ADM26615.1 & flavonoid 3\&apos;-hydroxylase & 5.395738 & -3.90333 & -2.00902 & 1.89431 \\
\hline
\end{tabular}



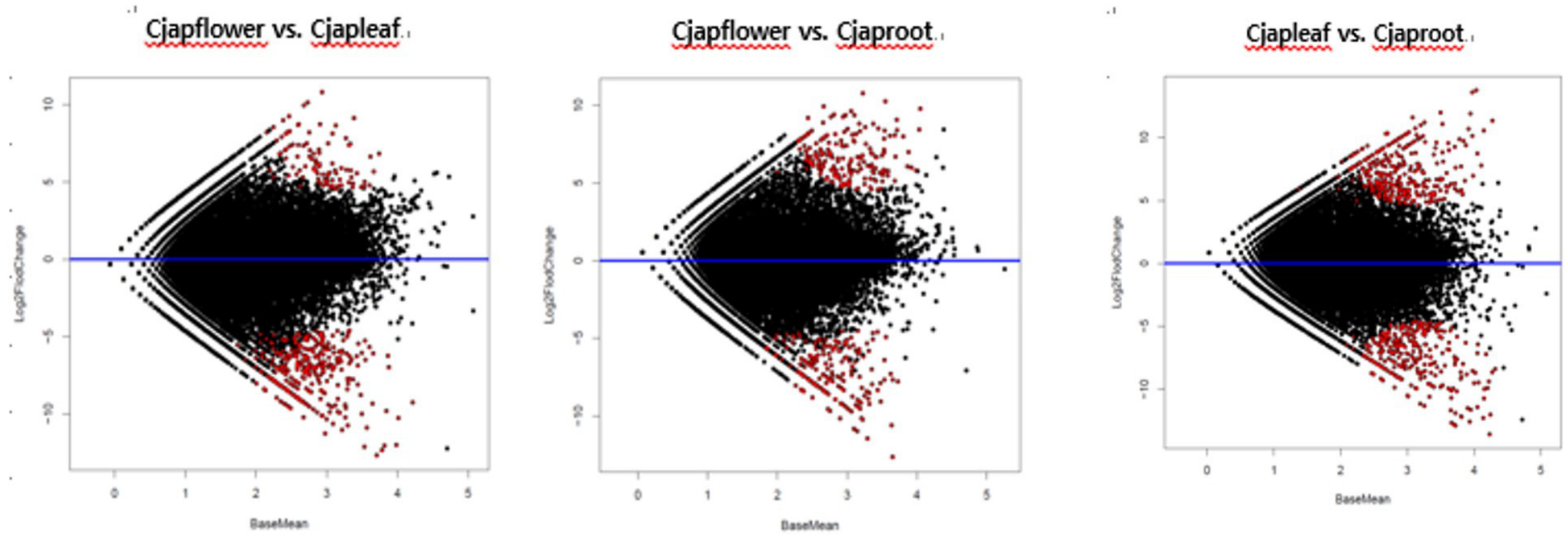

Supplementary Fig. 1. MA plot (log ratio vs. abundance plot) for flower vs. leaf, flower vs. root and leaf vs. root in Cirsium japonicum. 


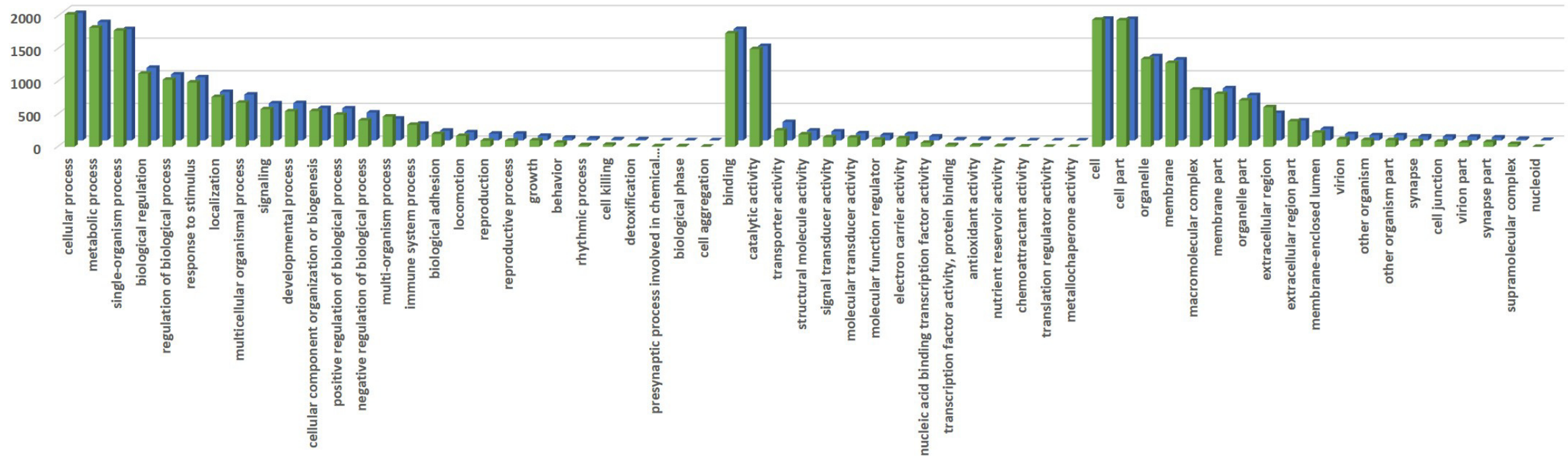

Supplementary Fig. 2. Comparison between up-regulated and down-regulated genes based on functional categories in Cirsium japonicum leaf and root tissues. 
UP @DOWN

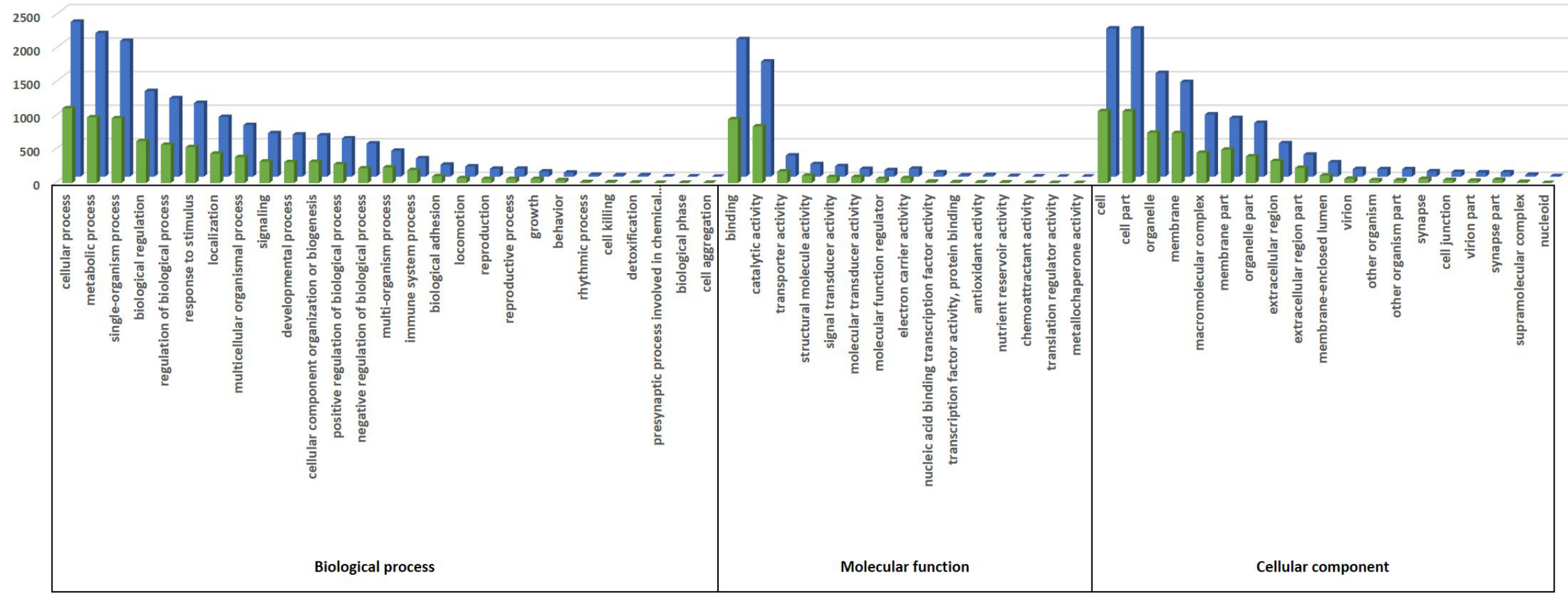

Cjapflower vs Cjaproot

Supplementary Fig. 3. 4-Comparison between up-regulated and down-regulated genes based on functional categories in in Cirsium japonicum flower and root tissues. 
-UP $\square$ DOWN

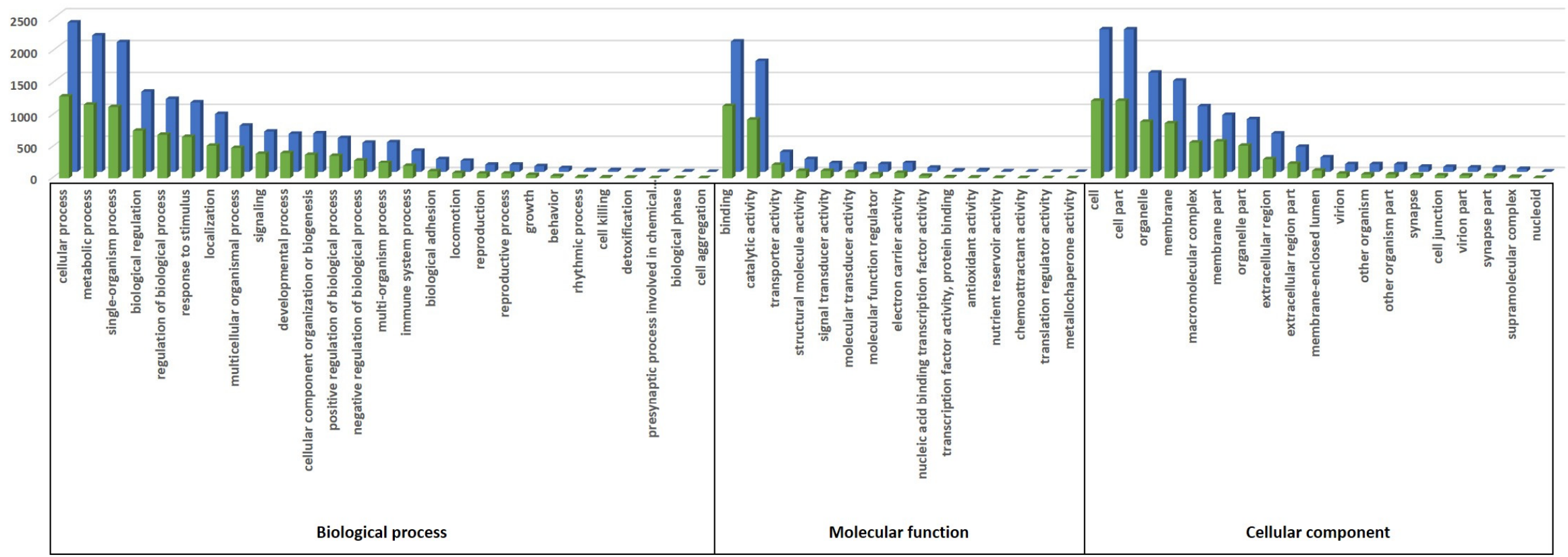

\section{Cjapflower vs Cjapleaf}

Supplementary Fig. 4. Comparison between up-regulated and down-regulated genes based on functional categories in in Cirsium japonicum flower and leaf. tissues 